\title{
EUS hepaticogastrostomy for bilioenteric anastomotic strictures: a permanent access for repeated ambulatory dilations? Results from a pilot study
}

Authors

Institution
Pablo Miranda-García, Jean M. Gonzalez, Juan I. Tellechea, Adrian Culetto, Marc Barthet

Gastroenterology Department. North Hospital, University of Mediterranean, Chemin des Bourrely, 13915 Marseille, France. Hopital Nord - Gastroenterology, Chemin des Bourrely, Marseille, France submitted:

2. November 2015

accepted after revision:

8. February 2016

\section{Bibliography}

DOI http://dx.doi.org/

10.1055/s-0042-103241

Published online: 30.3.2016

Endoscopy International Open

2016; 04: E461-E465

(c) Georg Thieme Verlag KG

Stuttgart · New York

E-ISSN 2196-9736

Corresponding author

Pablo Miranda-García,

\section{MD, PhD}

Gastroenterology Department.

North Hospital

University of Mediterranean

Chemin des Bourrely

13915 Marseille

France

Fax: +33-04-9196-8001

pmpablomiranda@gmail.com
Background and study aims: Postsurgical benign bilioenteric anastomotic strictures are a major adverse event of biliary surgery and endoscopic treatment, including endoscopic retrograde cholangiopancreatography (ERCP), is challenging in this setting. We present an innovative approach to treating this complication.

Patients and methods: Patients underwent endoscopic ultrasound (EUS)-hepaticogastrostomy (HG) to treat nonmalignant biliary obstructions. A first endoscopy was performed to create the hepaticogastrostomy and to drain the biliary tree. The second step had a therapeutic purpose: antegrade dilation of the anastomosis.

Results: Four men and three women with benign bilioenteric anastomotic strictures were included. Patients presented with jaundice or recurrent

\section{Introduction \\ $\checkmark$}

Postsurgical biliary strictures are a major adverse event of biliary surgery. Several invasive techniques including surgery, percutaneous drainage or transplantation have been attempted for management, with suboptimal results [1 -5].

EUS-guided biliary drainage (EUS-BD) has been dedicated to treatment of malignant biliary obstructions or as an alternative when the papilla is not endoscopically accessible $[6,7]$.

We report our experience in using EUS-BD to treat postsurgical benign biliary obstruction highlighting repeated antegrade dilation of the biliary stenosis through the hepaticogastrostomy (HG) and the use of double pigtail stents to maintain biliary drainage in a two-step approach. cholangitis. A fully covered HG stent was successfully deployed during the first endoscopy. During the second step, repeat antegrade dilation was performed through the HG in four cases (1-4 dilations) followed by double pigtail stenting in three cases. In three other patients, the stenosis was not crossable and a double pigtail stent was placed to maintain biliary drainage. All patients had symptom relief at the end of follow-up (45 weeks, range 33-64).

Conclusions: Dilation of anastomotic stenosis through a hepaticogastrostomy is feasible and may provide permanent biliary drainage or recurrent access to the biliary tree in patients with altered anatomy. Double pigtail stents might prevent migration.

\section{Patients and methods \\ $\nabla$}

Patients

Seven patients underwent a EUS-HG procedure to treat nonmalignant biliary obstructions in our hospital between August 2014 and September 2015. ERCP was impossible in all patients due to altered anatomy after previous gastrointestinal surgery: four patients had postsurgical stenosis from a hepatico-jejunal anastomosis, two patients had choledochal stenosis with an inaccessible duodenum, and in the remaining patient, ERCP was impossible ERCP because of a complete biliary defect at the liver hilum after right hepatectomy. All patients provided informed consent. This is a case-series retrospective study that was not registered as a clinical trial and was not considered for institutional review board approval.

\section{Endoscopic procedures}

Endoscopies were performed with the patients supine position and under general anesthesia, with endotracheal intubation by a single endoscopist. Prophylactic antibiotics were adminis- 


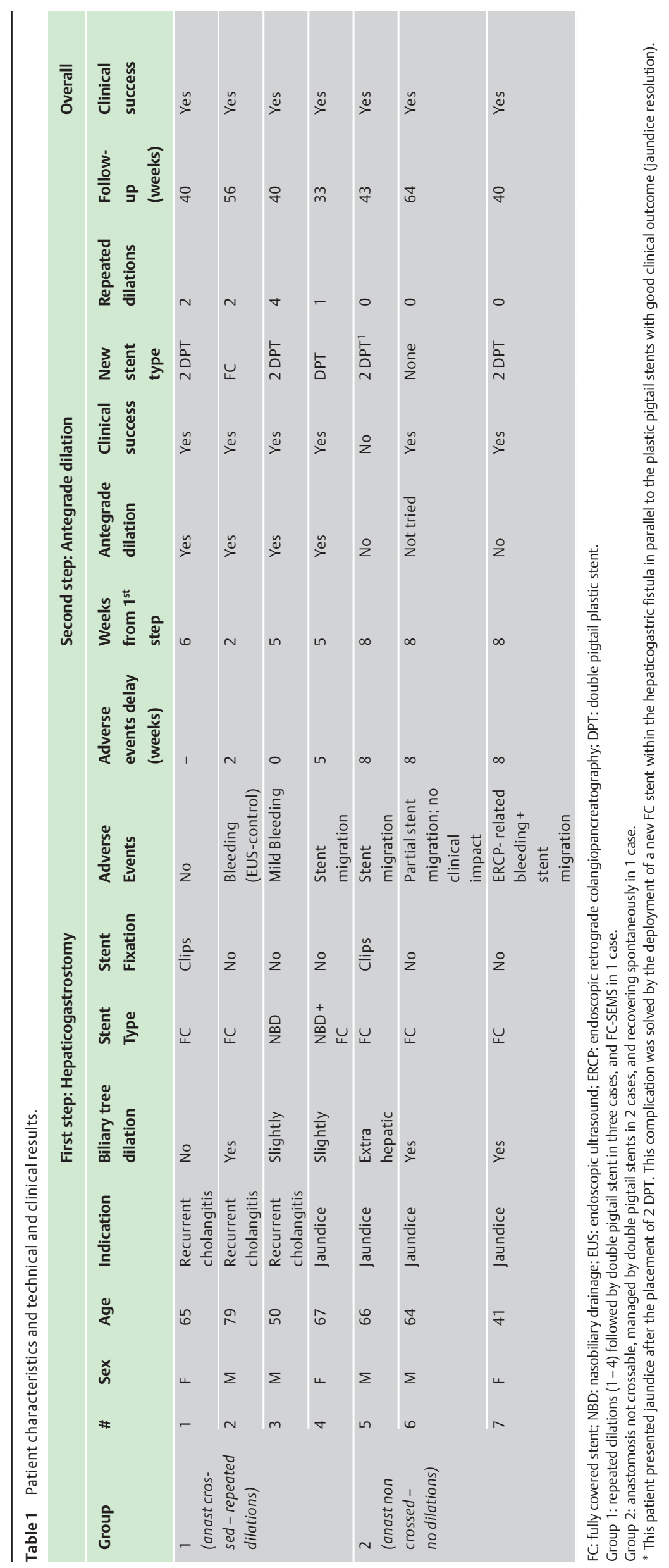




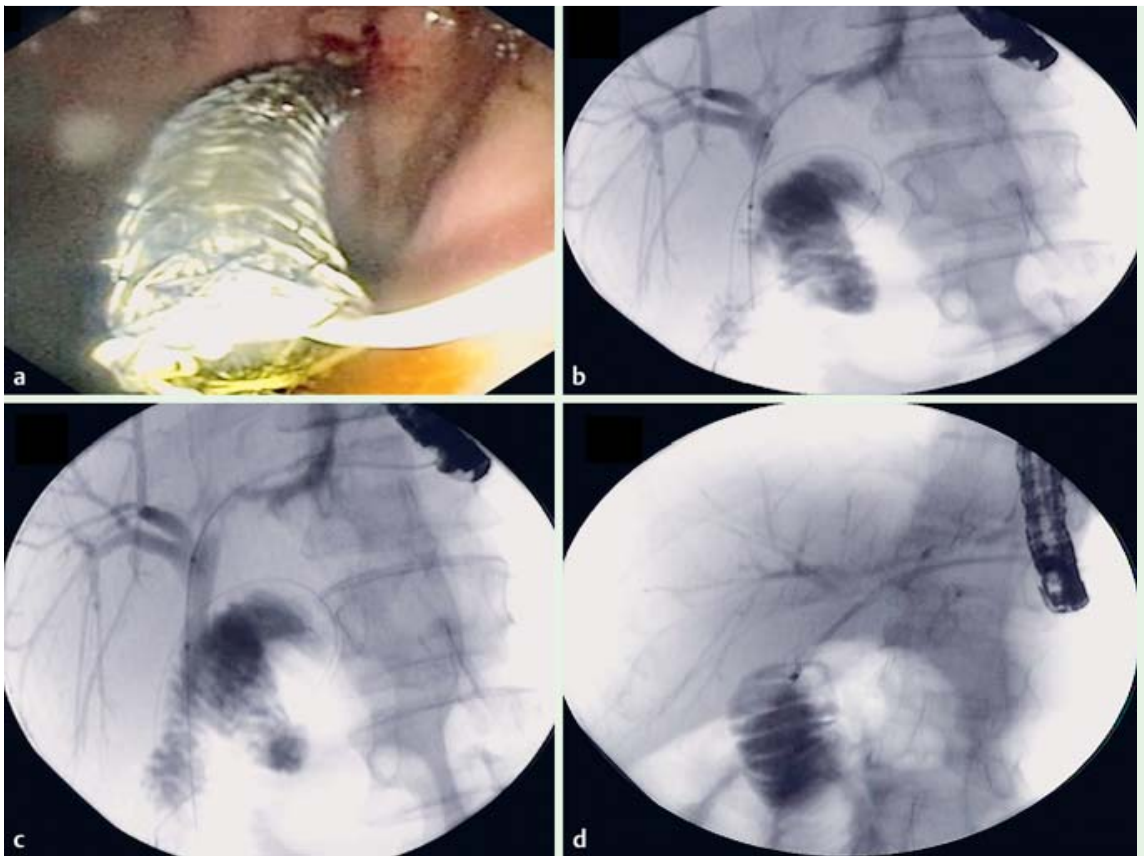

Fig. 1 a First endoscopic step. Endoscopic view of deployment of a fully covered metallic stent during hepaticogastrostomy. b Second endoscopic step. Fluoroscopic view of guidewire and dilation balloon being positioned within the hepaticojejunal anastomotic stricture. c Second endoscopic step. Fluoroscopic view of anastomosis dilation. Balloon inflated. $\mathbf{d}$ Second endoscopic step. Fluoroscopic view of deployment within the anastomotic stricture of a second double pigtail stent, with one end in the jejunum and the other end in the gastric lumen.

tered. A two-step approach, with the steps separated by 4 to 8 weeks, was planned for all patients.

During the first procedure, HG was performed according to the standardized technique, using a linear-array echoendoscope (Pentax EG 3670/Hitachi 550, Tokyo, Japan). The left biliary tree was reached through transgastric access. Diathermic dilation of access was performed using a 6-Fr cystostome (Endo-Flex Company, Germany). An 80-x 10-mm covered self-expendable metal stent (SEMS) (Taewoong Medical, Seoul, Korea) was placed between the biliary duct and the gastric lumen. In some cases, the stent was fixed to the gastric wall using Instinct clips (Cook Medical, Bloomington, IN) to prevent migration.

The second endoscopic step had a therapeutic purpose: antegrade dilation of the anastomotic stenosis. A guidewire (Jagwire: 0.035 inch, Boston Scientific, Natick, Mass, USA or Visiglide: 0.025 inch Olympus, Tokyo, Japan) was placed into the biliary tree through the HG stent or into the mature fistula in case of stent migration. Once the stenosis was crossed, dilation with an 8mm Max-Force balloon (Boston Scientific, Natick, Mass, USA) was performed. In some cases, biliary drainage was achieved using double pigtail plastic stents (Zimmon, Cook Medical, Bloomington, IN, USA) through the same hepaticogastric fistula in order to maintain patency, allowing further antegrade dilation. Double pigtail stents were left in place for a planned duration of 4 months.

\section{Results}

$\nabla$

Seven patients underwent EUS-HG for a nonmalignant indication. Patient characteristics and endoscopic features of our study are shown in $\bullet$ Table 1.

\section{First endoscopic step: hepaticogastrostomy}

A SEMS was successfully deployed in six cases ( $\bullet$ Fig. 1a). One patient was treated with naso-transgastric biliary drainage. In two patients, the stent was attached to the gastric wall with conventional clips in an attempt to decrease migration risk.
After this first step, clinical improvement was initially achieved in all patients. However, two patients had recurrence of jaundice due to stent migration before the second step ( 8 weeks).

\section{Second endoscopic step: antegrade dilation}

An antegrade therapeutic approach to the stenosis through the hepaticogastrostomy orifice was attempted after a median time of 5 weeks (range 2-9). During that phase, two clinical scenarios were encountered, depending on the ability to cross the stricture.

\section{Group 1 -anastomosis crossable}

In four patients, it was possible to pass the guidewire through the anastomosis, all of whom underwent one to four repeated dilations (5-16 weeks between dilations, mean 10 weeks) with an 8 -mm balloon ( $\bullet$ Fig. 1b, $\bullet$ Fig. 1b, $\bullet$ Fig. 1c). In three of these patients, each dilation procedure was followed by insertion of one or two double pigtail stents ( Fig. 1 ). One patient had two $10-$ Fr $15-\mathrm{cm}$ stents from the stomach to the jejunum; a second patient had a 7-Fr 10-cm and a 7-Fr 15-cm stent from the stomach to the jejunum; the third patient had one 7-Fr $10-\mathrm{cm}$ stent from the jejunum to the stomach. In one case the hepatico-gastric anastomosis was maintained patent with a fully covered metallic stent $(\bullet$ Table $\mathbf{1})$.

\section{Group 2 - anastomosis non crossable}

In three patients, it was not possible to pass through the stricture. Among them, two underwent, after removing the metallic stent, in an attempt to avoid migration, a double pigtail insertion (two 7-Fr $10-\mathrm{cm}$ plastic stents) to maintain patency of the hepaticogastric anastomosis. In the remaining patient, we observed stent migration into the stomach, but he no recurrence of jaundice or cholangitis $($ Table 1$)$.

The procedure was a technical success in four of seven patients success (57\%) and a clinical success in seven of seven patients (100\%), thanks to persistence of the HG. 


\section{Adverse events}

Three patients suffered from per-procedural bleeding during the first step.One case was immediately treated with EUS-guided embolization (2 cc of cyanoacrylate injection with a 19G needle) during the same endoscopy; in the second patient, the bleeding stopped spontaneously. The remaining patient had a hemoperitoneum related to the ERCP attempt (during catheterization) with injury of the right hepatic artery, which was successfully treated with radiological arterial embolization.

Post-procedural adverse events (AEs) consisted of three complete intragastric migrations, which were retreated with new biliary drainage through the same HG using double pigtail plastic stents, with both technical and clinical success. In one patient who had a partial migration, we decided not to perform the second step.

There were no severe AEs during the second endoscopic step. One patient had jaundice after placement of two plastic stents without migration. He was successfully treated with the addition of a fully covered SEMS ( $\bullet$ Table 1 ). No deaths related or unrelated to the procedure nor any incidents of cholangitis were recorded during follow-up (mean follow-up period: 45 weeks; range 33 $64)$.

\section{Discussion}

EUS-BD and antegrade endoscopic dilation have been well described for management of malignant biliary strictures, with an overall technical success rate of $87 \%$ and an $\mathrm{AE}$ rate of $27 \%$ for EUS-BD [7]. Conversely, application of this approach in management of benign obstructions has been poorly evaluated [6,8-15]. We present sequential management in patients who underwent EUS-HG for benign indications. The therapeutic strategy included two steps: First, EUS-HG was performed to create a sustainable hepaticogastric access; Second, an anterograde approach was employed to treat the cause of the obstruction. The two interventions were performed approximately 1 month apart so as to maintain fistula patency and reduce the risk of bile leakage and stent migration into the peritoneum during anastomosis dilation.

Regarding the first step, technical and clinical success was achieved in $100 \%$ of the cases. The perioperative AE rate was 28 $\%$ (two episodes of bleeding that required intervention), which was consistent with reports in the literature. Postoperatively, we encountered a high rate of stent migration (4/7) probably because the biliary tree was minimally dilated, which hindered good deployment of the stent, and also because we used fully covered stents to ensure that they could be removed. Nevertheless, stent migration was delayed (4-9 weeks), leaving enough time to create a reliable and mature HG fistula which drained the bile ducts with no consequences to patients and allowed for placement of double pigtail plastic stents instead of SEMS. That technique was effective in terms of both drainage and anti-migration, as evidenced by the single recurrence of jaundice and no incidents of migration.

During the second step, in four patients ( Table 1 ), we performed antegrade dilation that allowed repermeabilization of the anastomosis with technical success. Afterward, to keep the transgastric access patent, use of double pigtail plastic stents seemed to be a very interesting choice, reducing the risk of migration and maintaining the patency of the biliary fistula. Interestingly, we only had one AE during antegrade dilation and plastic stent deployment. Here, we propose a technique not previous- ly described in the literature, which could represent a new approach and way to calibrate anastomotic stenosis with multiple stenting when strictures cannot be reached with ERCP. Antegrade dilation was, however, successfully achieved in four out of seven patients (57\%), even if clinical success was optimal (100\%) due to persisting HG. These results should improve in future prospective and larger studies as we gain experience with this technique.

Indeed, we acknowledge that such an approach should be restricted to cases in which the transpapillary route cannot be used. However, in comparison to percutaneous drainage, this approach would permit internal drainage and reduce morbidity and the number of interventions. It could definitely restore normal biliary flow, either by achieving a permanent stricture calibration or a perennial hepaticogastric fistula, thus avoiding further interventions.

Our patients will undergo endoscopic procedures every 3 to 4 months over an estimated 18 months to dilate the anastomosis and proceed to iterative stricture calibration with the addition or exchange of plastic stents and we anticipate no anastomotic fibrosis after the dilations. This is the same as for regular ERCP management of postoperative bile duct injury. During the second step, double pigtail stents were used instead of SEMS to maintain patency of the HG and to reduce costs.

Our series does have several limitations. First, our sample was small and different types of benign strictures were included. Second, we encountered several complications during the procedure, but they were mostly related to the first step (hepaticogastrostomy formation) and not to the new technique we described here (antegrade dilation and anastomotic calibration with plastic stents). Nonetheless, stents are still in place in our patients and other complications could occur. In addition, follow-up of longterm outcome will be needed after stent retrieval in all patients to demonstrate permanent anastomotic stricture calibration. Indeed, in our patients, biliary drainage could be related to persistence of the HG fistula and not just recalibration of the anastomotic stricture and eventual improvement in antegrade biliary drainage. Finally, a double-balloon enteroscope could have been used as an alternative to biliary drainage in the setting of altered anatomy, but this technique is not available in our unit and it is technically challenging, even if it could be considered the gold standard. In our opinion, our approach could become another tool in management of a difficult clinical situation.

The authors would like to emphasize that this is a pilot study and our results should be regarded as preliminary data. Our technique evolved as we gained experience with the approach in each patient of our series. At this moment, we think that the best methodology could be to create a HG in the first step, with a fully covered metallic stent, and then proceed to scheduled stricture dilation through the HG fistula and calibration of the stricture with 7 to $10-\mathrm{Fr}, 15-\mathrm{cm}$ plastic double pigtail stents for 1 year. A prospective multicenter study of such an approach is being developed.

In conclusion, EUS-HG in the setting of benign bile duct strictures may provide permanent access to a bilioenteric anastomosis. Antegrade dilation of a bilioenteric anastomotic stricture through the HG is feasible and may result in more anatomic and permanent biliary drainage. The use of double pigtail stents may help achieve permanent stricture dilation and prevent stent migration.

Competing interests: None 


\section{References}

1 Rothlin MA, Lopfe M, Schlumpf $R$ et al. Long-term results of hepaticojejunostomy for benign lesions of the bile ducts. Am J Surg 1998; 175 $22-26$

2 Genest JF, Nanos E, Grundfest-Broniatowski S et al. Benign biliary strictures: an analytic review (1970 to 1984). Surgery 1986; 99: 409-413

3 Tocchi A, Costa G, Lepre L et al. The long-term outcome of hepaticojejunostomy in the treatment of benign bile duct strictures. Ann Surg 1996; 224: $162-167$

4 Smith AC, Dowsett JF, Russell RCG et al. Randomised trial of endoscopic steriting versus surgical bypass in malignant low bile duct obstruction. Lancet 1994; 344: 1655 - 1660

5 Günther $R W$, Schild $H$, Thelen M. Percutaneous transhepatic biliary drainage: experience with 311 procedures. Cardiovasc Intervent Radiol 1988; 11: 65-71

6 Poincloux L, Rouquette O, Buc E et al. Endoscopic ultrasound-guided biliary drainage after failed ERCP: cumulative experience of 101 procedures at a single center. Endoscopy 2015; 47: 794-801

7 Iwashita T, Doi S, Yasuda I. Endoscopic ultrasound-guided biliary drainage: a review. Clin J Gastroenterol 2014; 7: 94-102

8 Iwashita T, Yasuda I, Doi S et al. Endoscopic ultrasound-guided antegrade treatments for biliary disorders in patients with surgically altered anatomy. Dig Dis Sci 2013; 58: 2417-2422
9 Bories E, Pesenti C, Caillol $F$ et al. Transgastric EUS-guided biliary drainage. Endoscopy 2007; 39: 287-291

10 Püspök A, Lomoschitz F, Dejaco C. Endoscopic ultrasound guided therapy of benign and malignant biliary obstruction: a case series. Am J Gastroenterol 2005; 100: 1743-1747

11 Artifon EL, Safatle-Ribeiro AV, Ferreira FC et al. EUS-guided antegrade transhepatic placement of a self-expandable metal stent in hepaticojejunal anastomosis. JOP 2011; 12: 610-613

12 Park do H, Jang JW, Lee SS et al. EUS-guided transhepatic antegrade balloon dilation for benign bilioenteric anastomotic strictures in a patient with hepaticojejunostomy. Gastrointest Endosc 2012; 75: 692 - 693

13 Park do DH, Jeong SU, Lee BU et al. Prospective evaluation of a treatment algorithm with enhanced guidewire manipulation protocol for EUS-guided biliary drainage after failed ERCP (with video). Gastrointest Endosc 2013; 78: 91 - 101

14 Shah JN, Marson F, Weilert F et al. Single-operator, single-session EUSguided anterograde cholangiopancreatography in failed ERCP or inaccesible papilla. Gastrointest Endosc 2012; 75: 56-64

15 Will U, Thieme A, Fueldner F et al. Treatment of biliary obstruction in selected patients by endoscopic ultrasonography (EUS)-guided transluminal biliary drainage. Endoscopy 2007; 39: 292 - 295 